\title{
The Condition of Ni-Cr Alloy Surface After Abrasive Blasting with Various Parameters
}

\author{
Weronika Czepułkowska, Emilia Wołowiec-Korecka, and Leszek Klimek
}

\author{
(Submitted August 5, 2019; in revised form September 27, 2019; published online October 25, 2019)
}

\begin{abstract}
The parameters of abrasive blasting process directly affect the condition of the metal surface, changing the degree of surface roughness and wettability, depending on the size of the used particle, the pressure or type of abrasive. The aim of this study was to analyze the condition of $\mathrm{Ni}-\mathrm{Cr}$ alloy surface after abrasive blasting using various process variants. The samples were blasted by $\mathrm{Al}_{2} \mathrm{O}_{3}$ abrasive using various particle sizes and pressures of the process. Basic and specific roughness parameters were investigated, and a surface wettability test was performed, and the percentage share of abrasive particles penetrated in the surface after abrasive blasting was also examined. The most considerable differences in the condition of the surface were observed with the change in the particle size of $\mathrm{Al}_{2} \mathrm{O}_{3}$. Statistical analysis confirmed the statistical significance of all these relationships.
\end{abstract}

Keywords abrasive blasting, fixed ceramic restorations, $\mathrm{Ni}-\mathrm{Cr}$ alloy, prosthetics, surface condition

\section{Introduction}

Abrasive blasting is a widely used process that has many applications. Among other things, it concerns the preparation of surfaces for all coatings, paints and varnishes. Due to the action of abrasive under high pressure on the surface, it is possible to remove any contaminants such as paint residues and rust from it. At the same time, the adhesive properties of surface increase (Ref 1). Abrasive blasting is used in medicine and dentistry to increase the surface area of implants or dental restorations (Ref 2-4). When creating ceramic crowns and prosthetic bridges, substructures made of dental alloys, such as cobalt-chromium or nickel-chromium alloy, are subjected to abrasive blasting $\left(\mathrm{Al}_{2} \mathrm{O}_{3}, \mathrm{SiC}\right)$ in order to ensure adequate conditions on the metal surface to filling with semiliquid ceramic during firing. Due to the creation of a connection between materials with very different properties, the analysis of mechanisms determining its durability is extremely important.

The surface condition of the material subjected to abrasive blasting is dependent on several factors. The properties of the processed material have an important role, with particular regard to its hardness or the presence of any impurities or

This article is an invited submission to JMEP selected from presentations at The XXII Physical Metallurgy and Materials Science Conference: Advanced Materials and Technologies (AMT 2019) held June 9-12, 2019, in Bukowina Tatrzańska, Poland, and has been expanded from the original presentation.

Weronika Czepułkowska, Emilia Wołowiec-Korecka, and Leszek Klimek, Institute of Materials Science and Engineering, Faculty of Mechanical Engineering, Lodz University of Technology, Stefanowskiego Street 1/15, 90-924 Lodz, Poland. Contact e-mails: weronika.czepulkowska@p.lodz.pl, emilia.wolowiec@p.lodz.pl, and leszek.klimek@p.lodz.pl. oxides on the surface. The surface condition is also influenced by the machining parameters and abrasive material used during the process (Ref 5). The pressure, angle of the nozzle or its distance from the surface of the material used can affect the roughness or the number of abrasive particles penetrated into its surface. However, depending on its hardness or shape, the abrasive also affects the appearance of the treated surface. Ultimately, the surface after abrasive blasting is the result of these factors (Ref 6).

The first and basic feature of the surface, which is the result of abrasive blasting, is the appearance of roughness. It is one of several components of the geometric surface structure, arising inter alia as a result of affecting the surface of different treatments with varying parameters (Ref 7$)$. Its description uses parameters that characterize specific profile features. The creation of suitable increases in the surface area allows the covering material to anchor into the resulting unevenness. Depending on the used abrasive blasting parameters, such as abrasive type (particle size, hardness), machining pressure or even angle between the material and the nozzle, different surface properties are obtained.

The degree of increasing the surface area of the materials directly affects its wettability and, hence, the adhesive properties. By adhesion, it is meant the interaction of particles of substances in contact with each other, which is why the surface free energy test (SFE) is often used to. To determine the energy, the contact angle is measured, and based on its value, the total surface energy and its components are calculated (Ref 1, 8, 9). The surface condition can be inferred from both SFE values and wetting angles. In the case of prosthetics, in the first contact with the treated metal surface, the ceramic is a mixture of powder and water, so its nature is polar. When increasing the temperature in the furnace, the water evaporates and its character changes to apolar; therefore, the analysis of the surface condition of the metal should include tests of the parameters described above.

Another direct result of abrasive blasting is the penetration of abrasive particles into the surface. Their quantity depends on the parameters used during the process, where, in the case of the cobalt-chromium alloy, the share of aluminum oxide is present, from approx. 5 to $30 \%$ of the surface (Ref 10). The 
value of this, as well as the previous parameters described, is also affected by the hardness of the material undergoing the processes. The hard material will be more resistant to the impact of abrasive compared to soft material. Among the most commonly used alloys in prosthetics, the $\mathrm{Co}-\mathrm{Cr}$ and $\mathrm{Ni}-\mathrm{Cr}$ alloys are distinguished, where the former is a material of higher hardness, and for this, there are tests related to the state of the surface after sandblasting for the connection with dental ceramics (Ref 11). There is a significant influence of the parameters of the blasting process and the surface condition of the metal on the quality of the metal-ceramic connection; therefore, the work was subjected to nickel-chromium alloy tests, for which there are no literature data on the study of the dependence of surface condition on sandblasting. The aim of the work was determined, which is the analysis of the impact of variable parameters of abrasive blasting processes on roughness, SFE and the percentage of abrasive particles penetrated of the nickel-chromium alloy.

\section{Materials and Methods}

A total of 18 cylindrical samples with a diameter of $7.8 \mathrm{~mm}$ and a height of $15 \mathrm{~mm}$, made of a nickel-chromium alloy (Heraenium $^{\circledR}$ NA, Heraeus Kulzer), were blasted (Alox 2001, Effegi Brega) using abrasive $\mathrm{Al}_{2} \mathrm{O}_{3}$ according to the parameters presented in Table 1. The angle of the sandblaster nozzle to the surface of the material was $45^{\circ}$, and the distance from the nozzle to the surface was $15 \mathrm{~mm}$. The processing time for each element was $20 \mathrm{~s}$. The samples after blasting were subjected to cleaning in an ultrasonic bath (Emmi-55HC-Q, Emag) using deionized water for $8 \mathrm{~min}$ and then dried with compressed air.

The surface roughness test was carried out using a HommelEtamic T8000 profilometer (Jenoptik) with a measuring probe with a radius of $0.005 \mathrm{~mm}$. The length of the profile mapping was $400 \mu \mathrm{m}$, and the measurement speed was set at $0.5 \mathrm{~mm} / \mathrm{s}$. Three profiles were made for each surface, from which $\mathrm{Ra}, \mathrm{Rz}$, $\mathrm{Rk}$, Rsm, $V_{0}$ and $L_{\mathrm{r}}$ roughness parameters were determined according to PN-EN ISO 4288:2011 norm (Ref 12). The wettability test was performed by dropping polar and dispersion liquid on the metal surface using the FM40 EasyDrop device (Krüss GmbH Germany). On the basis of the measurement of contact angles with measuring liquids formed on the surface of the alloy, according to the Owens-Wendt equation, surface free energy (SFE) was determined (Ref 8). Another study was the analysis of the surface of the metal alloy in terms of the amount of abrasive particles penetrated into it. For this purpose, photographs taken from the scanning electron microscope, which were binarized, were used. Using the ImageJ $1.48 \mathrm{v}$ program (Wayne Rasband), the percentage of area covered by the abrasive was calculated. Elementary mapping was created using EDS microanalysis.

Table 1 Parameters of the processes

\begin{tabular}{lccc}
\hline & \multicolumn{3}{c}{ Pressure, kPa } \\
\cline { 2 - 4 } Particle size, $\boldsymbol{\mu m}$ & $\mathbf{2 0 0}$ & $\mathbf{4 0 0}$ & $\mathbf{6 0 0}$ \\
\hline 50 & $\left(\mathrm{Al}_{2} \mathrm{O}_{3}\right)_{50,2}$ & $\left(\mathrm{Al}_{2} \mathrm{O}_{3}\right)_{50,4}$ & $\left(\mathrm{Al}_{2} \mathrm{O}_{3}\right)_{50,6}$ \\
110 & $\left(\mathrm{Al}_{2} \mathrm{O}_{3}\right)_{110,2}$ & $\left(\mathrm{Al}_{2} \mathrm{O}_{3}\right)_{110,4}$ & $\left(\mathrm{Al}_{2} \mathrm{O}_{3}\right)_{110,6}$ \\
250 & $\left(\mathrm{Al}_{2} \mathrm{O}_{3}\right)_{250,2}$ & $\left(\mathrm{Al}_{2} \mathrm{O}_{3}\right)_{250,4}$ & $\left(\mathrm{Al}_{2} \mathrm{O}_{3}\right)_{250,6}$ \\
\hline
\end{tabular}

The results of the measurements were analyzed to verify the following statistical hypotheses: 1 . Are there statistically significant differences in mean values of roughness parameters between groups?2. Are there statistically significant differences in the mean values of wettability or surface free energy between groups?

\section{Results}

\subsection{Surface Roughness}

Figure 1 shows the topography of the alloy surface after abrasive blasting with $50-\mu \mathrm{m}$ particle size and $600 \mathrm{kPa}$ pressure. The results of tests of $\mathrm{Ra}, \mathrm{Rz}$ and $\mathrm{Rk}$ parameters indicate a linear, directly proportional dependence of the value of these parameters on the particle size of the abrasive, in all pressure variants (Fig. 2 and 3). The Kruskal-Wallis test showed that for pressures of 400 and $600 \mathrm{kPa}$, the differences between the mean $\mathrm{Ra}$ and $\mathrm{Rz}$ values between groups 50 and $250 \mu \mathrm{m}$ are statistically significant $(\alpha=0.05)$. For $110-\mu \mathrm{m}$ particle and $200 \mathrm{kPa}$ pressure, statistically significant differences are not observed.

The results of the roughness parameters Rsm, $V_{0}$ and $L_{\mathrm{r}}$ increase as the particle size increases, in all pressure variants (Fig. 3 and 4). In statistical tests (Kruskal-Wallis test), it was not shown that these are statistically significant differences $(\alpha=0.05)$.

\subsection{Wettability}

The results of the surface wettability test prove that both for the obtained angles from wetting the surface with polar and dispersion liquid, no significant differences are observed (Fig. 5 and 6). In the case of SFE statistically significant differences, $(\alpha=0.05)$ was observed for the particle size of $250 \mu \mathrm{m}$ between the groups where the pressure was 200 and $600 \mathrm{kPa}$. The highest SFE value was observed at $600 \mathrm{kPa}$ and 250- $\mu \mathrm{m}$ particle (Fig. 7).

\subsection{Amount of Abrasive Particles Penetrated into the Surface}

Analysis of the percentage share of abrasive particles penetrated into the surface shows the effect of particle size on

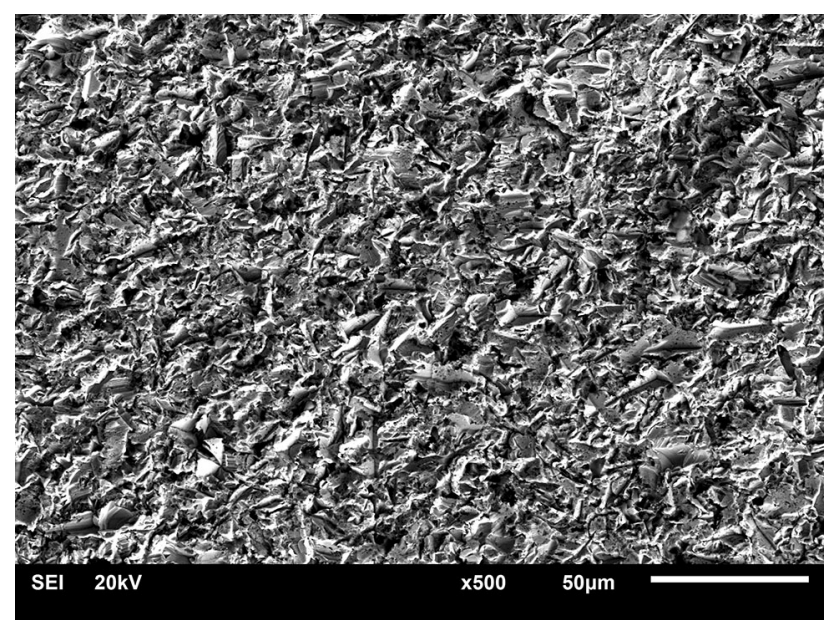

Fig. 1 SEM photography of nickel-chromium alloy surface after abrasive blasting with $50-\mu \mathrm{m}$ particle size and $600 \mathrm{kPa}$ pressure 

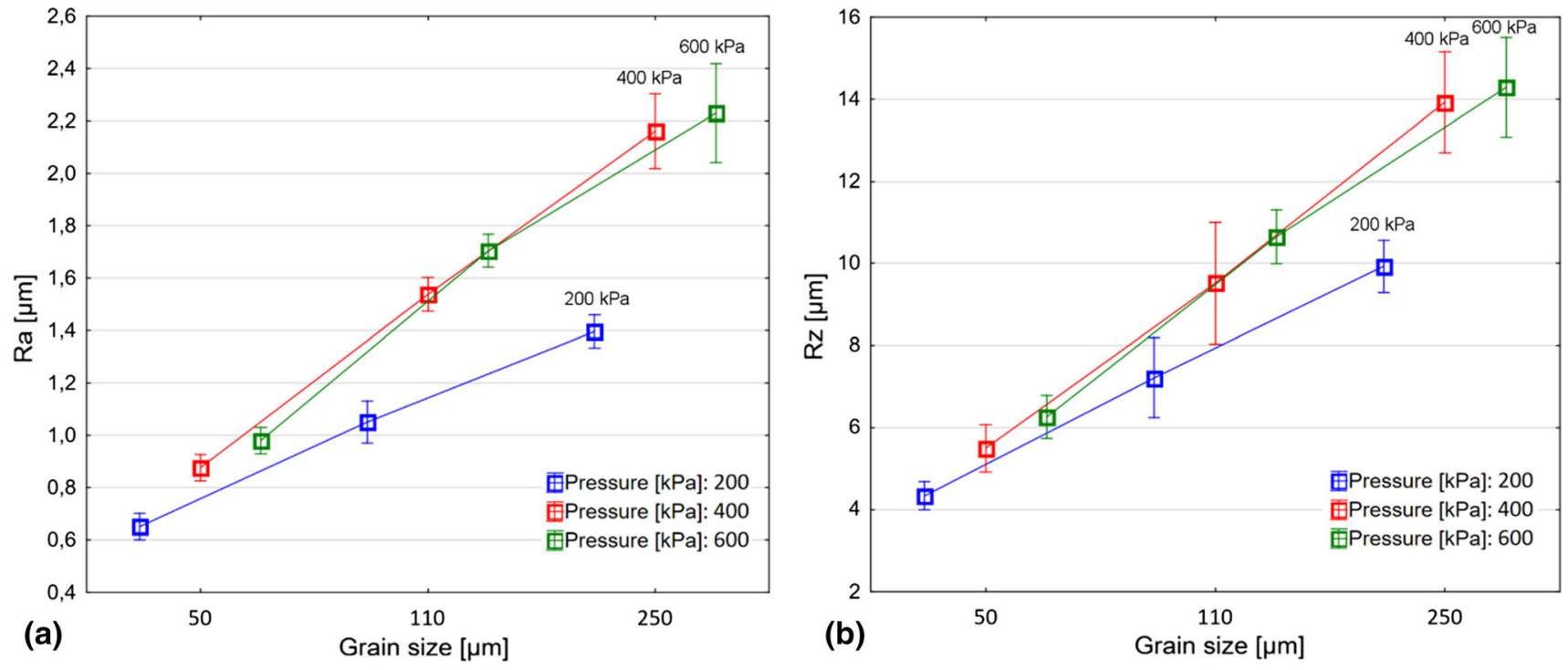

Fig. 2 Graph of mean values of Ra and Rz parameters grouped on the particle size $(\mu \mathrm{m})$ and categorized against pressure $(\mathrm{kPa})$
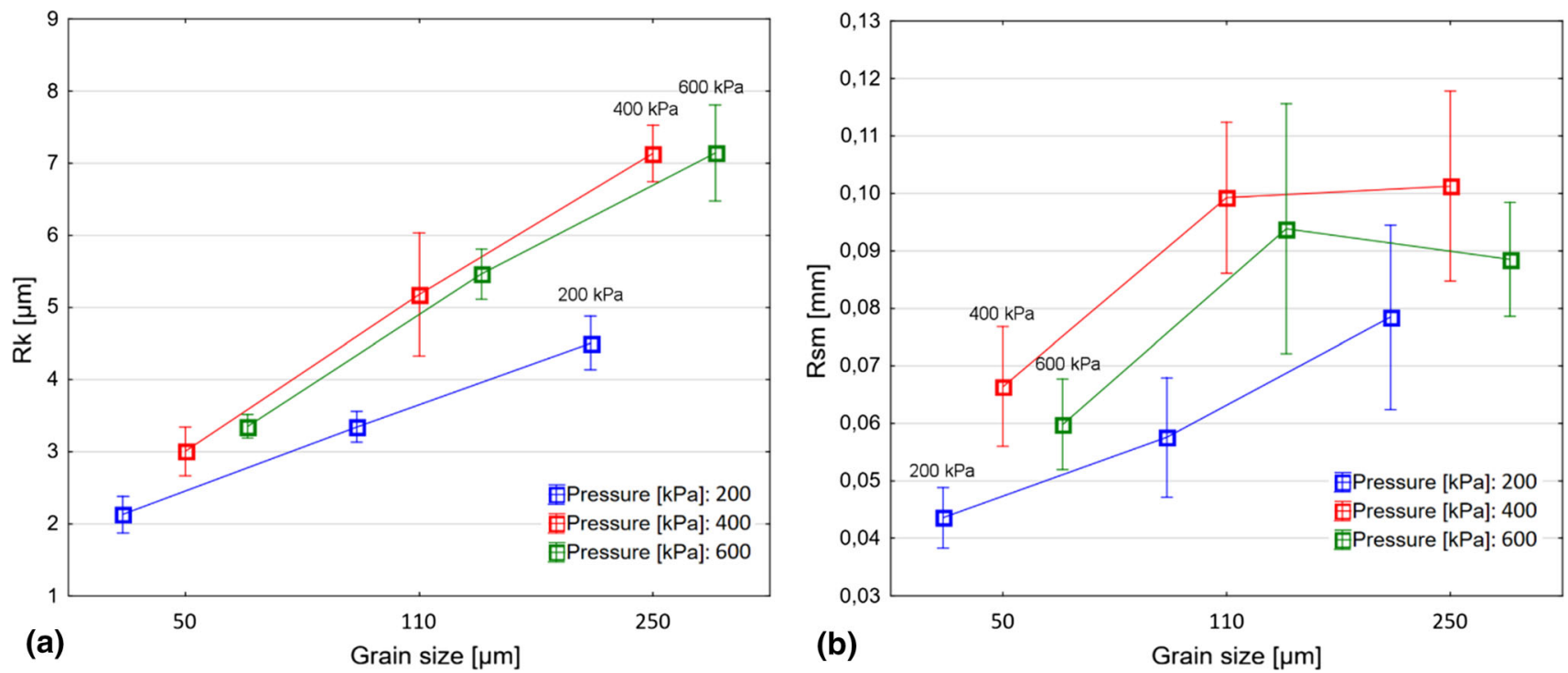

Fig. 3 Graph of mean values of Rk and Rsm parameters grouped on the particle size $(\mu \mathrm{m})$ and categorized against pressure $(\mathrm{kPa})$

the number of penetrate particles. The largest amount of penetrated particles was observed for the smallest particle, in all the tested pressures (Fig. 8). For the pressures of 200 and $600 \mathrm{kPa}$, significant differences were observed between the particle sizes of 50 and $250 \mu \mathrm{m}$ (Kruskal-Wallis test, $\alpha=0.05$ ). At a pressure of $400 \mathrm{kPa}$, significant differences occurred between the $50-\mu \mathrm{m}$ particle and the 110 - and $250-\mu \mathrm{m}$ particles. Figure 9 shows the elementary mapping of alloy surface. The places with penetrated abrasive particles are visible, where $\mathrm{Al}$ and $\mathrm{O}$ elementary particles are present.

\section{Discussion}

The study of basic roughness parameters most frequently reported in the literature such as $\mathrm{Ra}, \mathrm{Rz}$ or Rk showed a similar relationship between the used particle size and the pressure of the working medium on their values (Fig. 2 and 3). It turns out that the use of pressure 400 and $600 \mathrm{kPa}$ does not significantly affect the roughness, compared with the pressure of $200 \mathrm{kPa}$, where a slight reduction in these values is observed. This can be explained by the fact that at low pressures $(200 \mathrm{kPa})$ of the medium, sufficient cutting values have not yet been achieved. This happened at $400 \mathrm{kPa}$ pressure, and further increase in pressure did not cause major changes. There is a certain limit value of roughness that can be obtained at a given particle size, and it was achieved at a pressure of about $400 \mathrm{kPa}$. Further increasing the pressure can only increase the material removal rate, without altering its roughness parameters. The size of used the abrasive has a much more significant influence on the roughness parameters. It is observed, what is rather obvious, much higher values for the largest particles (Ref 13). A similar relationship was received by Pietnicki et al. and Gołębiowski et al. in their research, but in this case, cobalt-chromium alloy roughness and titanium roughness were analyzed (Ref 14, 15). 

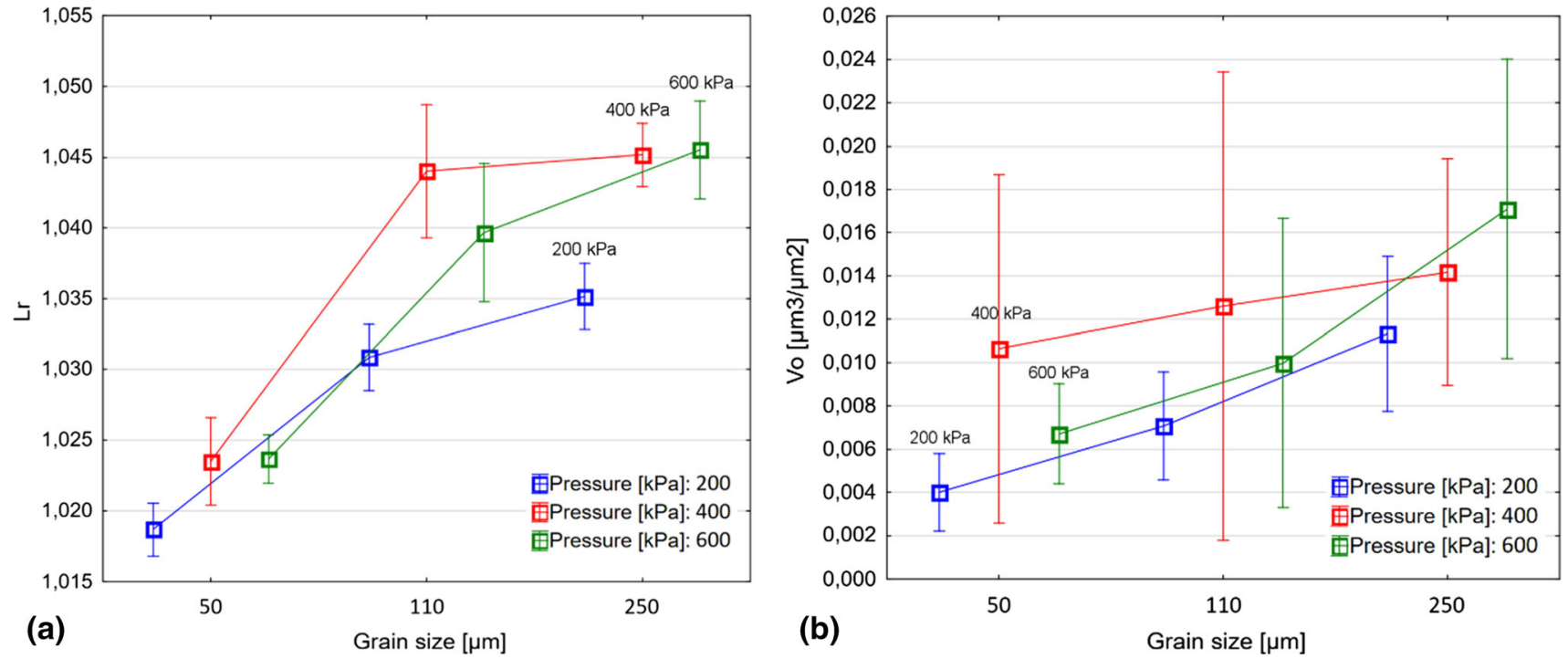

Fig. 4 Graph of mean values of $L_{\mathrm{r}}$ and $V_{0}$ parameters grouped on the particle size $(\mu \mathrm{m})$ and categorized against pressure $(\mathrm{kPa})$
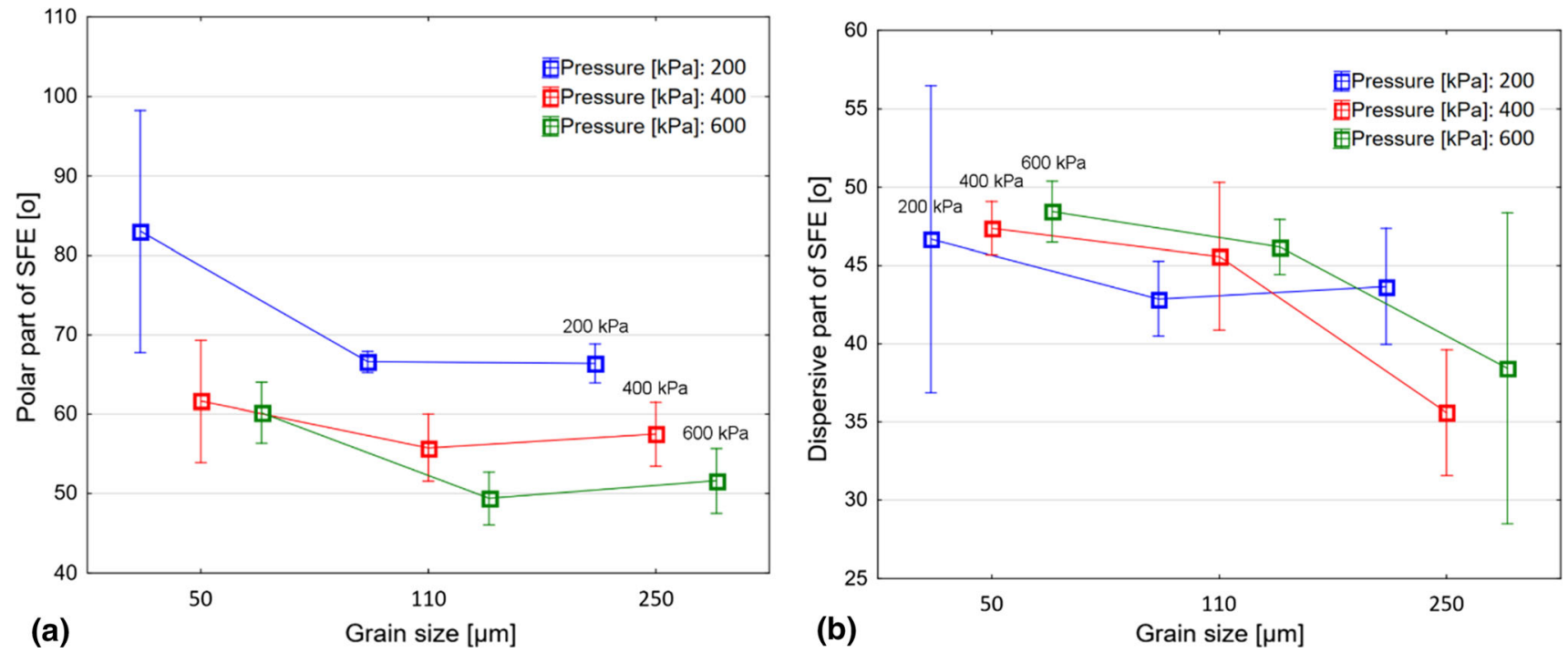

Fig. 5 Graph of mean values of polar and dispersion angles, grouped on the particle size $(\mu \mathrm{m})$ and categorized against pressure $(\mathrm{kPa})$

Analysis of only these three parameters may be insufficient to describe the surface condition of the tested material, in terms of preparing the surface for firing ceramics. These are only height parameters, and they do not give information about the shape and distances between irregularities; however, this has a very large impact on the possibility of the ceramics to cover the unevenness and mechanical attachment in them. Therefore, in the research other parameters were measured, which in our opinion more accurately describe the increase in the surface area. Guided by their selection, we made a few analogies. First of all, we adopted the similarity of the fired ceramic to the paint coatings, and hence, the parameters $V_{0}$ (retention volume) and $L_{\mathrm{r}}$ (profile length ratio) appeared. The possibility of the ceramics to cover the unevenness depends, to a large extent, on the distance between them and hence the Rsm parameter. The results for Rsm, $L_{\mathrm{r}}$ and $V_{0}$ show a similar relationship between increasing the values and larger abrasive particles, although for these parameters a significant standard error of the mean is observed (Fig. 3 and 4). These results cause that there are no differences with statistical significance. Summing up the above examinations, it can be concluded that the larger the particle (at some optimal pressure), the greater the unevenness and more favorable surface preparation. However, it seems that there is some optimum roughness, because on the one hand the greater the roughness, the better covered with ceramics and deeper attachment. But on the other, the smaller the roughness, the higher the amount fastenings.

Other surface parameters relevant to the preparation of the ceramic surface are wettability and surface free energy. It is known that the better the wetting of the surface with liquid ceramic, the easier it will cover the unevenness. On the other hand, the surface free energy gives information about the activity of this surface and the possibility of making connections. The polar contribution of SFE for the surface of the nickel-chromium alloy decomposes in a similar arrangement as the values of the roughness parameters, where the highest results are obtained for the surface after treatment with pressures of 400 and $600 \mathrm{kPa}$ (Fig. 6). In comparison with 

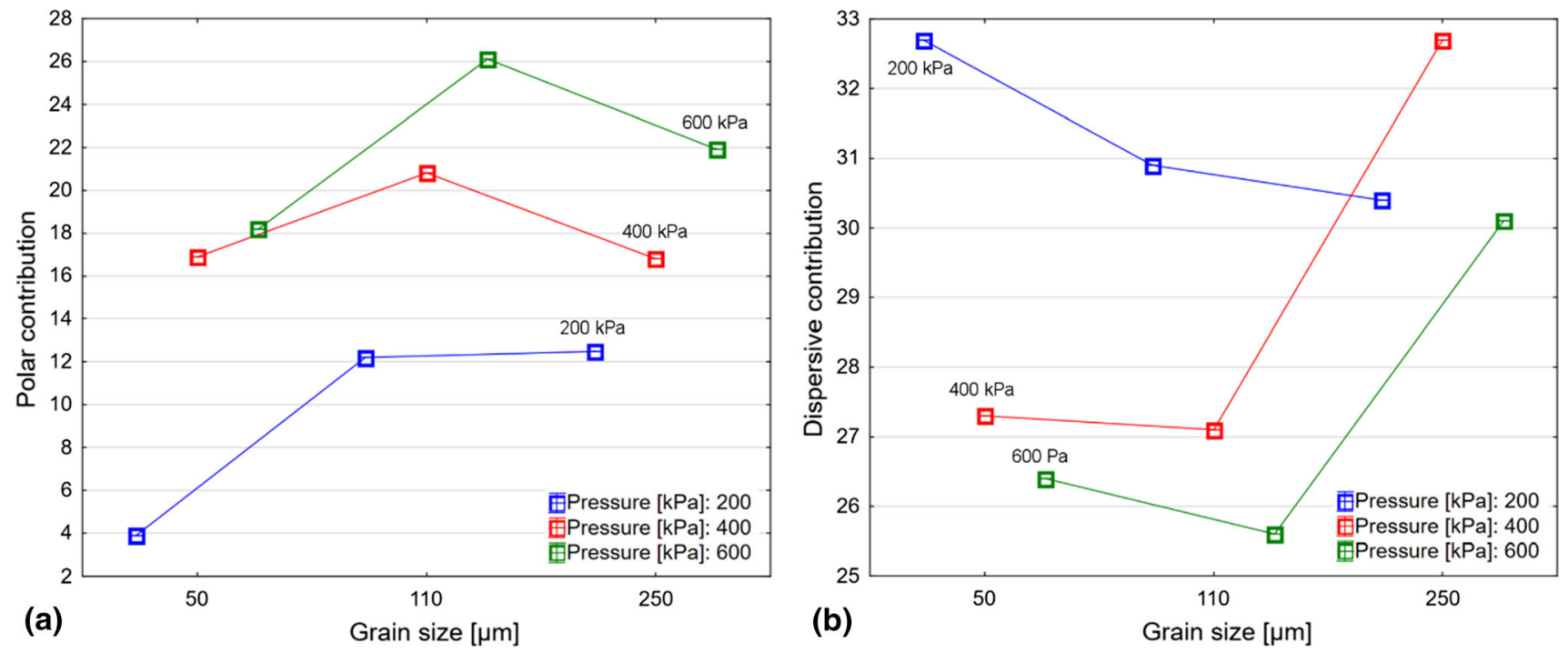

Fig. 6 Graph of mean values of polar and dispersion contribution of SFE, grouped on the particle size ( $\mu$ m) and categorized against pressure $(\mathrm{kPa})$

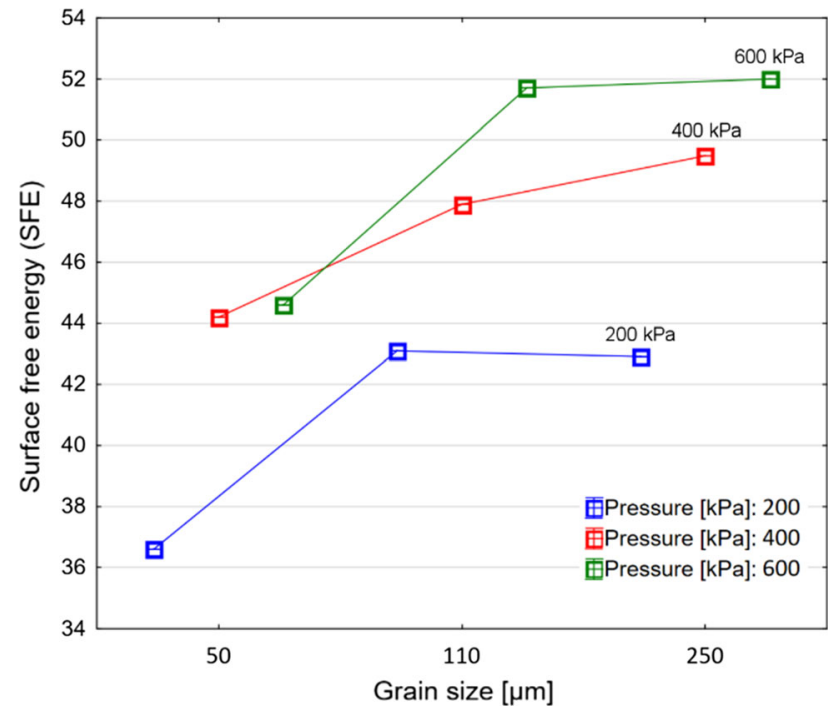

Fig. 7 Graph of mean SFE values grouped on the particle size $(\mu \mathrm{m})$ and categorized against pressure $(\mathrm{kPa})$

the other pressures, much higher values of dispersion contribution of SFE are observed for the pressure of $200 \mathrm{kPa}$ and the particle size of 50 and $110 \mu \mathrm{m}$. For the cobalt-chromium alloy, the results of the SFE with a much lower value are obtained. For surface treated with abrasive size $250 \mu \mathrm{m}$, under pressure $400 \mathrm{kPa}$ the value of SFE is $27.8 \mathrm{~mJ} / \mathrm{m}^{2}$ and for alloy Ni-Cr is equal to $49.5 \mathrm{~mJ} / \mathrm{m}^{2}$ (Ref 14). Titanium for the same abrasive blasting parameters reaches the $33.2 \mathrm{~mJ} / \mathrm{m}^{2} \mathrm{SFE}$ value (Ref 15). Finally, it can be observed that the value of surface free energy is affected by the hardness of the material and the surface roughness. The analysis of the results of total free energy shows that for pressures of 400 and $600 \mathrm{kPa}$ it is similar for the particles of 110 and $250 \mu \mathrm{m}$ and is definitely higher than for the blasting with $50-\mu \mathrm{m}$ particle size. From the viewpoint of surface activity, therefore, two gradations of particles should be preferred. However, if we take into account the other components of SFE, polar and dispersive energy, the polar contribu-

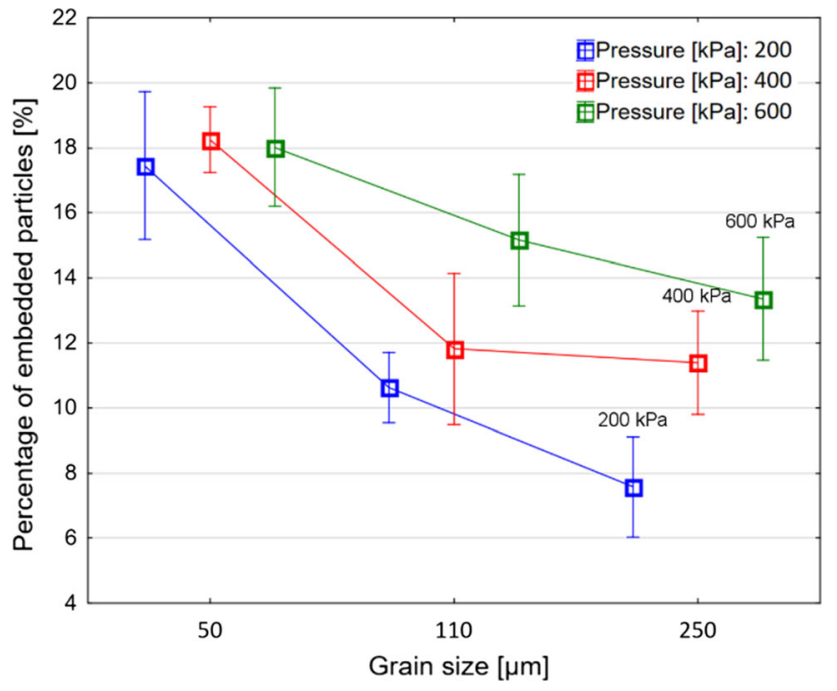

Fig. 8 Graph of the average amounts of penetrated particles into the surface after abrasive blasting, grouped on the particle size $(\mu \mathrm{m})$ and categorized against pressure $(\mathrm{kPa})$

tion of SFE achieves the highest value for particle processing of $250 \mu \mathrm{m}$, and the dispersion is the highest for particle processing of $110 \mu \mathrm{m}$. The polar contribution of SFE will only be important in the initial phase of applying the ceramic when it is suspended in water. During heating to firing temperature, the water is removed. After melting, the liquid ceramic is an apolar liquid, and at the time of its firing this contribution of SFE will be of decisive importance. It seems, therefore, that the $110-\mu \mathrm{m}$ particle is optimal for abrasive blasting.

The consequence of the abrasive blasting is the abrasive particles penetrated into the treated surface. For the pressures of $200 \mathrm{kPa}$ and $600 \mathrm{kPa}$, significant differences in the number of penetrated particles between the groups with the used particle size of 50 and $250 \mu \mathrm{m}$ were demonstrated. This dependence can also be significant for a pressure of $400 \mathrm{kPa}$. A smaller amount of penetrated particles, observed for the largest abrasive, may be caused by its crumbling during contact with 


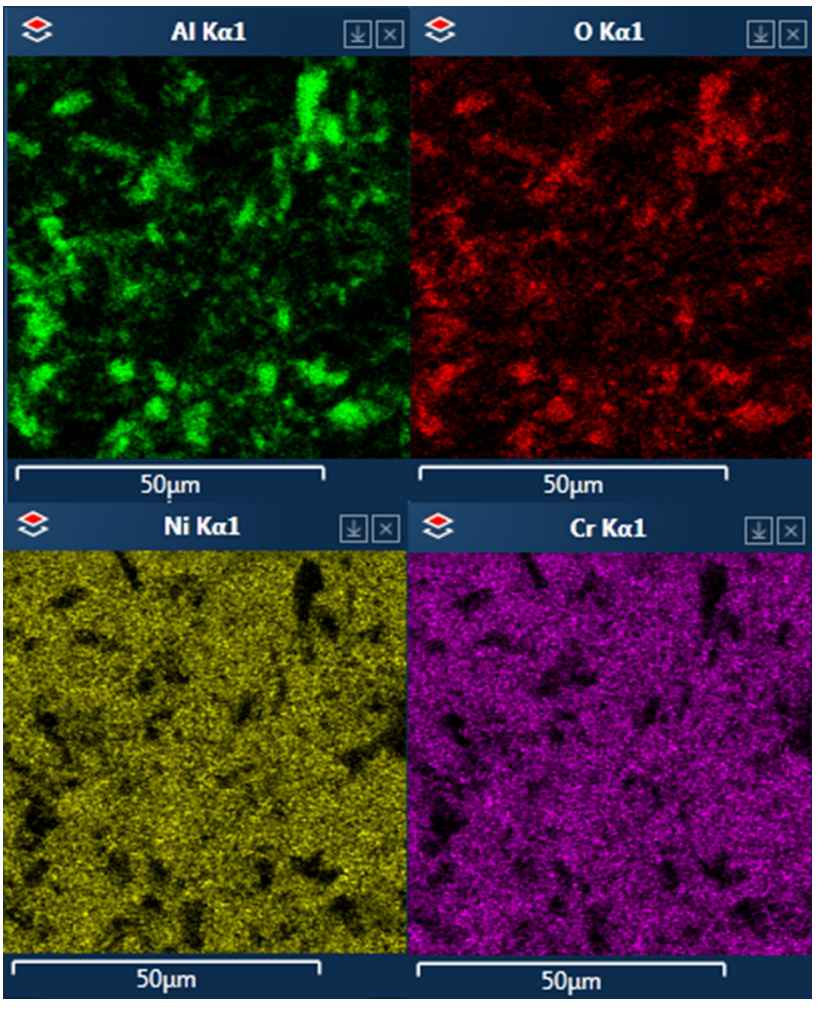

Fig. 9 Elemental mapping of the nickel-chromium alloy surface after abrasive blasting with $50-\mu \mathrm{m}$ particle size and $600 \mathrm{kPa}$ pressure

the surface or difficulty in driving through too large particle diameter. Small abrasive is lighter, so at the nozzle outlet at the same working pressure it can reach higher speeds, which, at its smaller size, allows more effective penetration into the metal surface and anchoring. The same dependence of the amount of penetrated particle on the size is also observed for other materials (Ref 10, 15). From a practical point of view, the amount of abrasive particles penetrated in the surface may be of significant importance for the subsequent behavior of the ceramic-fired ceramic during the use of the prosthetic restoration. The presence of the second phase can accelerate corrosion. In addition, penetrated particles, due to their sharp edges, can cause stress concentrate and initiate ceramic cracking.

In summary, the surface condition of the nickel-chromium alloy after blasting is mainly influenced by the size of abrasive particle used. The machining pressure used results in a significant difference only when using the lowest tested value, which is $200 \mathrm{kPa}$.

\section{Conclusion}

The following conclusions were made on the basis of the research:

1. There is a linear, directly proportional dependence of the $\mathrm{Ra}, \mathrm{Rz}, \mathrm{Rk}$ roughness parameters on the abrasive particle size. Differences between $\mathrm{Ra}$ and $\mathrm{Rz}$ values for the particle size of 50 and $250 \mu \mathrm{m}$ at pressures of 0.4 and $0.6 \mathrm{MPa}$ are statistically significant.
2. The size of the particle used and the machining pressure have no significant effect on the parameters of Rsm, $V_{0}$ and $L_{\mathrm{r}}$.

3. The use of larger particle abrasive significantly reduces the amount of particles driven into the surface of the NiCr alloy.

4. The treatment pressure has a significant effect on the total surface energy.

\section{Open Access}

This article is distributed under the terms of the Creative Commons Attribution 4.0 International License (http://creativeco mmons.org/licenses/by/4.0/), which permits unrestricted use, distribution, and reproduction in any medium, provided you give appropriate credit to the original author(s) and the source, provide a link to the Creative Commons license, and indicate if changes were made.

\section{References}

1. A. Rudawska, I. Danczak, M. Müller, and P. Valasek, The Effect of Sandblasting on Surface Properties for Adhesion, Int. J. Adhes. Adhes., 2016, 70, p 176-190

2. H.B. Kara, E. Dilber, O. Koc, A.N. Ozturk, and M. Bulbul, Effect of Different Surface Treatments on Roughness of IPS Empress 2 Ceramic, Lasers Med. Sci., 2012, 27(2), p 267-272

3. G.D. Byrne, L. O’Neill, B. Twomey, and D.P. Dowling, Comparison Between Shot Peening and Abrasive Blasting Processes as Deposition Methods for Hydroxyapatite Coatings Onto a Titanium Alloy, Surf. Coat. Technol., 2013, 216, p 224-231

4. A. Grübl, A. Kolb, G. Reinisch, G. Fafilek, G. Skrbensky, and R. Kotz, Characterization, Quantification, and Isolation of Aluminum Oxide Particles on Grit Blasted Titanium Aluminum Alloy Hip Implants, $J$. Biomed. Mater. Res. B Appl. Biomater., 2007, 83B(1), p 127-131

5. A. Momber, Blast Cleaning Technology, Springer, New York, 2008, p 7-54

6. J. Brezinová, A. Guzanová, and D. Draganovská, Abrasive Blast Cleaning and Its Application, Trans Tech Publications, Stafa-Zurich, 2015

7. Standard, PN-EN 828:2013-05, 2013

8. D.K. Owens and R.C. Wendt, Estimation of the Surface Free Energy of Polymers, J. Appl. Polym. Sci., 1969, 13(8), p 1741-1747

9. A. Tsujimoto, W. Barkmeier, T. Takamizawa, M. Latta, and M. Miyazaki, The Effect of Phosphoric Acid Pre-etching Times on Bonding Performance and Surface Free Energy with Single-Step SelfEtch Adhesives, Oper. Dent., 2016, 41(4), p 441-449

10. K. Pietnicki, E. Wołowiec, and L. Klimek, Modeling of the Number of Stubble Stuck Elements After Abrasive Jet Machining-Processing, Arch. Foundry Eng., 2011, 11(3), p 51-54

11. K. Pietnicki, E. Wołowiec, and L. Klimek, The Effect of Abrasive Blasting on the Strength of a Joint Between Dental Porcelain and Metal Base, Acta Bioeng. Biomech., 2014, 16(1), p 63-68

12. Standard, PN-EN ISO 4288:2011, 2011

13. T. Külünk, M. Kurt, Ç. Ural, Ş. Külünk, and I.S. Baba, Effect of Different Air-Abrasion Particles on Metal-Ceramic Bond Strength, J. Dent. Sci., 2011, 6(3), p 140-146

14. K. Pietnicki, Influence of abrasive blasting parameters on the quality of the combination of dental ceramics with cobalt-chromium alloys. Ph.D. Thesis, Lodz University of Technolgy, Łódź, 2013 (in Polish)

15. M. Gołębiowski, E. Wołowiec, and I.L. Klimek, Airborne-Particle Abrasion Parameters on the Quality of Titanium-Ceramic Bonds, J. Prosthet. Dent., 2015, 113(5), p 453-459

Publisher's Note Springer Nature remains neutral with regard to jurisdictional claims in published maps and institutional affiliations. 